\title{
CHARACTERIZATION OF FUNCTIONS IN TERMS OF RATE OF CONVERGENCE OF A QUADRATURE PROCESS
}

\author{
Q. I. RAHMAN AND G. SCHMEISSER
}

(Communicated by J. Marshall Ash)

\begin{abstract}
We discuss the relationship between the regularity properties of a continuous $2 \pi$-periodic function $f$ and the behavior of the remainder of the trapezoidal rule on $[0,2 \pi]$ applied to $f$.
\end{abstract}

\section{INTRODUCTION}

It is well known that certain classes of periodic functions can be characterized in terms of the degree of best approximation by trigonometric polynomials. To illustrate this, we quote the following results.

Theorem A $[10$, p. 61]. A continuous $2 \pi$-periodic function $g$ is infinitely differentiable if and only if

$$
\lim _{n \rightarrow \infty} n^{k} E_{n}^{T}(g)=0
$$

for every exponent $k$. Here

$$
E_{n}^{T}(g)=\inf \sup _{x \in \mathbf{R}}\left|g(x)-t_{n}(x)\right|,
$$

where the infimum is taken over all trigonometric polynomials $t_{n}$ of degree at most $n$.

Theorem B [10, p. 61]. The property

$$
E_{n}^{T}(g)=O\left(n^{-k-1}\right), \quad k \text { an integer } \geq 0,
$$

is a necessary and sufficient condition that the continuous $2 \pi$-periodic function $g$ is $k$ times differentiable and the $k$ th derivative belongs to the Zygmund class $\mathscr{Z}$.

In this paper we wish to investigate whether classes of periodic functions can be similarily characterized in terms of the speed of convergence of a quadrature

Received by the editors June 1, 1989 and, in revised form, January 8, 1990.

1980 Mathematics Subject Classification (1985 Revision). Primary 41A55; Secondary 41A25, $46 \mathrm{E} 10,65 \mathrm{D} 32$.

Key words and phrases. Quadrature formula, trapezoidal rule, speed of convergence, regularity properties, function spaces. 
procedure. We treat this question for the trapezoidal rule

$$
\int_{0}^{2 \pi} f(x) d x=\frac{2 \pi}{n} \sum_{\nu=1}^{n} f\left(\frac{2 \pi \nu}{n}\right)+R_{n}[f],
$$

where $f$ belongs to $C_{2 \pi}(\mathbf{R})$, the space of continuous $2 \pi$-periodic functions $f: \mathbf{R} \rightarrow \mathbf{C}$. Our choice is motivated by the fact that for periodic functions this rule is optimal in certain ways. For example it is the "best" quadrature formula of the form

$$
\int_{0}^{2 \pi} f(x) d x=\sum_{\nu=1}^{n} w_{\nu} f\left(x_{\nu}\right)+\rho_{n}[f]
$$

for the Sobolev space $W_{L^{2}}^{k}$ of $2 \pi$-periodic functions (see Definition 2 below) where the weights $w_{\nu}$ and the nodes $0<x_{1}<\cdots<x_{n} \leq 2 \pi$ are arbitrary (see [12, Theorem 3]).

We start with certain observations that will shed some light on the problem under consideration and will also motivate the formulation of the desired characterizations.

Let $\mathscr{T}_{k}$ be the space of all trigonometric polynomials of degree at most $k$. Using the well-known fact that $\sum_{\nu=0}^{n-1} \omega^{\nu}=0$ for an $n$th root of unity $\omega \neq 1$, we obtain

$$
R_{n}[f]=0 \text { for all } n>k
$$

and all $f \in \mathscr{T}_{k}$. However, (2) does not characterize $\mathscr{T}_{k}$ since (2) also clearly holds for any function $f \in C_{2 \pi}(\mathbf{R})$ which is odd on $[0,2 \pi]$; i.e., $f(x) \equiv$ $-f(2 \pi-x)$. It may even hold if $f$ is not of the form $t+\varphi$ where $t \in \mathscr{T}_{k}$ and $\varphi$ is odd on $[0,2 \pi]$. In fact, the function

$$
g(x)=\sum_{n=1}^{\infty} \frac{\mu(n)}{n} e^{i n x}
$$

considered by C. H. Ching (see [1, 9]) wherein $\mu$ is the Möbius function, has the remarkable property that $R_{n}[g]=0$ for all $n \in \mathbf{N}$ although $g+c$ is not odd on $[0,2 \pi]$ for any constant $c$. This shows that the property (2) does not contain "much" information about the function $f$. Broadly speaking, the remainder is small if the function either has strong regularity properties or else possesses certain symmetries. We note that the regularity properties of a function $f$ are inherited by its translates

$$
f_{h}: x \mapsto f(x+h), \quad h \in \mathbf{R},
$$

but the same is not true for the symmetries. Since we are interested in regularity properties, it seems natural to us to involve translates of $f$ into our consideration. We shall in fact prove the following:

Theorem 1. Let $f \in C_{2 \pi}(\mathbf{R})$. Then $f \in \mathscr{T}_{k}$ if and only if $R_{n}\left[f_{h}\right]=0$ for all $n>k$ and $|h| \leq \pi / n$.

Certain other spaces of functions can also be characterized via $R_{n}\left[f_{h}\right]$. 
Definition 1. Let $B_{2 \pi, d}^{2}$ denote the set of all $2 \pi$-periodic functions $f$ holomorphic in

$$
S_{d}:=\{z \in C:|\Im z|<d\}
$$

and satisfying

$$
\|f\|:=\sup _{|y|<d}\left(\frac{1}{2 \pi} \int_{0}^{2 \pi}|f(x+i y)|^{2} d x\right)^{1 / 2}<+\infty .
$$

As usual we shall denote by $\ell^{2}$ the Hilbert space of sequences $\left(a_{n}\right)_{n \in \mathbf{N}}$ for which $\sum_{n \in \mathbf{N}}\left|a_{n}\right|^{2}<\infty$.

Theorem 2. For $f \in C_{2 \pi}(\mathbf{R})$, the following statements are equivalent:

(i) $f$ is the restriction to $\mathbf{R}$ of a function belonging to $B_{2 \pi, d}^{2}$,

(ii) $\left|R_{n}\left[f_{h}\right]\right| \leq b_{n} e^{-n d}$ for all $n \in \mathbf{N}$ and $|h| \leq \pi / n$, where $d$ is a positive number and $\left(b_{n}\right)_{n \in \mathbf{N}} \in \ell^{2}$.

Definition 2. Let $W_{L^{2}}^{k}$ be the space of functions (Sobolev space) $f: \mathbf{R} \rightarrow \mathbf{C}$ of period $2 \pi$ for which $f^{(k-1)}$ exists and is absolutely continuous with $f^{(k)} \in$ $L^{2}(0,2 \pi)$.

Theorem 3. For $f \in C_{2 \pi}(\mathbf{R})$, the following statements are equivalent:

(i) $f \in W_{L^{2}}^{k}$ for some integer $k \geq 2$,

(ii) $R_{n}\left|\left[f_{h}\right]\right| \leq b_{n} n^{-k}$ for all $n \in \mathbf{N}$ and $|h| \leq \pi / n$ where $\left(b_{n}\right)_{n \in \mathbf{N}} \in \ell^{2}$.

From the proof of Theorem 3, it will be clear that (ii) implies (i) for $k=1$ as well. However, (i) does not imply (ii) in the case $k=1$, as the next theorem shows.

Theorem 4. There exists a function $f \in W_{L^{2}}^{1}$ such that

$$
\sum_{n \in \mathbf{N}} n^{2}\left|R_{n}\left[f_{h}\right]\right|^{2}=\infty
$$

It has been pointed out by Professor C. K. Chui through the editor that our results have some relationship with those in [2] and [4-7] but it seems to us that there is no overlap. In order to illustrate the connection to those papers we quote the following:

Theorem $\mathbf{C}$ [5, Theorem 1]. Let $f$ be a function holomorphic in the open unit disk with $f^{\prime} \in H^{p}, 1<p \leq \infty$, and

$$
\frac{1}{n} \sum_{k=1}^{n} f(\exp (i 2 \pi k / n))=0
$$

for each $n=1,2, \ldots$. Then $f$ is the zero function.

Theorem D [4, Theorem 1]. Denote by $B^{s}$, where $s>0$, the class of periodic functions $f$ with period one whose Fourier coefficients satisfy $f^{\wedge}(n)=O\left(n^{-s}\right)$ 
and define

$$
S_{\alpha, n}(f):=\frac{1}{n} \sum_{k=1}^{n} f\left(\alpha+\frac{k}{n}\right), \quad R_{\alpha, n}(f):=S_{\alpha, n}(f)-\int_{0}^{1} f(t) d t .
$$

Let $\alpha$ and $\beta$ be any two real numbers in $[0,1]$.

(i) Suppose $\alpha-\beta$ is irrational and $f$ is in $B^{s}$ with $s>1$ such that $S_{\alpha, n}(f)=S_{\beta, n}(f)=0$ for all $n>0$. Then $f$ is the zero function.

(ii) Suppose $\alpha-\beta$ is rational. Then there is a trigonometric function $g \neq 0$ such that $S_{\alpha, n}(g)=S_{\beta, n}(g)=0$.

(iii) Suppose $\alpha-\beta$ is irrational. Then there exist trigonometric polynomials $P_{\alpha, m}$ such that

$$
\begin{aligned}
& R_{\beta, n}\left(P_{\alpha, m}\right)=S_{\beta, n}\left(P_{\alpha, m}\right)=0, \\
& R_{\alpha, n}\left(P_{\alpha, m}\right)=S_{\alpha, n}\left(P_{\alpha, m}\right)=\delta_{m, n},
\end{aligned}
$$

for all positive integers $m$ and $n$.

\section{Auxiliary Results}

We start by recalling the following classical result of L. Fejér.

Lemma 1. Let $f \in C_{2 \pi}(\mathbf{R})$. If

$$
f^{\wedge}(\mu):=\frac{1}{2 \pi} \int_{0}^{2 \pi} f(x) e^{-i \mu x} d x, \quad \mu \in \mathbf{Z},
$$

then uniformly for all $x \in \mathbf{R}$ we have

$$
\lim _{m \rightarrow \infty} \sum_{\mu=-m}^{m}\left(1-\frac{|\mu|}{m+1}\right) f^{-}(\mu) e^{i \mu x}=f(x) .
$$

We use Lemma 1 to obtain a representation for $R_{n}\left[f_{h}\right]$.

Lemma 2. If $f \in C_{2 \pi}(\mathbf{R})$, then uniformly for all $h \in \mathbf{R}$ we have

$$
\lim _{m \rightarrow \infty}-2 \pi \sum_{|j| \leq[m / n]}^{\prime}\left(1-\frac{|j n|}{m+1}\right) f^{-}(j n) e^{i j n h}=R_{n}\left[f_{h}\right] .
$$

Here and in the rest of the paper, the prime indicates that the term corresponding to the value 0 of the index is omitted in the summation.

Proof. By the result of Fejerr,

$$
f_{h}(x)=f(x+h)=\lim _{m \rightarrow \infty} \sum_{\mu=-m}^{m}\left(1-\frac{|\mu|}{m+1}\right) f^{-}(\mu) e^{i \mu(x+h)}
$$

uniformly for $x, h \in \mathbf{R}$. Hence

$$
\begin{aligned}
R_{n}\left[f_{h}\right]= & \lim _{m \rightarrow \infty} \sum_{\mu=-m}^{m}\left(1-\frac{|\mu|}{m+1}\right) f^{-}(\mu) \\
& \times\left\{\int_{0}^{2 \pi} e^{i \mu(x+h)} d x-\frac{2 \pi}{n} \sum_{\nu=1}^{n} e^{i \mu(h+2 \pi \nu / n)}\right\} .
\end{aligned}
$$


Since $\int_{0}^{2 \pi} e^{i \mu(x+h)} d x$ is equal to $2 \pi$ or 0 depending on whether $\mu$ is equal to zero or not, and $\frac{1}{n} \sum_{\nu=1}^{n} e^{i 2 \pi \mu \nu / n}$ is equal to 1 or 0 depending on whether $\mu$ is a multiple of $n$ or not, we obtain (4).

The next lemma contains a characterization of the class $B_{2 \pi, d}^{2}$ which represents an important step toward the proof of Theorem 2.

Lemma 3. A function $f \in C_{2 \pi}(\mathbf{R})$ is the restriction to $\mathbf{R}$ of a function belonging to $B_{2 \pi, d}^{2}$ if and only if $f^{\wedge}(n)=a_{n} e^{-|n| d}$ where $\left(a_{ \pm n}\right)_{n \in \mathbf{N}} \in \ell^{2}$.

Proof. Let $f \in B_{2 \pi, d}^{2}$, and set

$$
\alpha_{n}(y):=\frac{1}{2 \pi} \int_{0}^{2 \pi} f(x+i y) e^{-i n x} d x \text { for } n \in \mathbf{Z} .
$$

Contour integration of $f(z) e^{-i n z}$ along the rectangle with vertices $0,2 \pi$, iy, and $2 \pi+i y$, where $|y|<d$ and $n y<0$, yields

$$
\begin{aligned}
f^{\wedge}(n) & =\frac{1}{2 \pi} \int_{0}^{2 \pi} f(x) e^{-i n x} d x \\
& =\frac{1}{2 \pi} \int_{0}^{2 \pi} f(x+i y) e^{-i n(x+i y)} d x=e^{-|n y|} \alpha_{n}(y),
\end{aligned}
$$

since the contributions coming from the two vertical segments cancel each other out because of the assumed periodicity. Using (3), Bessel's inequality shows that

$$
\sum_{n \in \mathbf{Z}}\left|f^{\wedge}(n)\right|^{2} e^{-2|n y|}=\sum_{n \in \mathbf{Z}}\left|\alpha_{n}(y)\right|^{2} \leq\|f\|^{2} \text { for } y \in(-d, d) .
$$

Hence, setting $a_{n}:=f^{\wedge}(n) e^{|n| d}$, we obtain

$$
\sum_{n \in \mathbf{Z}}\left|a_{n}\right|^{2} e^{-2|n|(d-|y|)} \leq\|f\|^{2} \text { for } y \in(-d, d),
$$

which implies that $\sum_{n \in \mathbf{Z}}\left|a_{n}\right|^{2} \leq\|f\|^{2}$.

Conversely, if $f^{\wedge}(n)=a_{n} e^{-|n| d}$, where $\left(a_{ \pm n}\right)_{n \in \mathbf{N}} \in \ell^{2}$, then the function $f$ has a uniformly and absolutely convergent Fourier series, and $f$ may be extended to $S_{d}$ by the holomorphic function

$$
f(x+i y):=\sum_{n=-\infty}^{\infty} f^{-}(n) e^{i n(x+i y)}
$$

which, by Parseval's relation, satisfies

$$
\frac{1}{2 \pi} \int_{0}^{2 \pi}|f(x+i y)|^{2} d x=\sum_{n=-\infty}^{\infty}\left|f^{-}(n) e^{-n y}\right|^{2} \leq \sum_{n=-\infty}^{\infty}\left|a_{n}\right|^{2} e^{-2|n|(d-|y|)}
$$

for all $y \in(-d, d)$. Consequently, $\|f\|<\infty$ and $f \in B_{2 \pi, d}^{2}$.

For the proof of Theorem 4, we need to recall a notation and a result about infinite matrices. 
Definition 3 [8, p. 303]. An infinite symmetric matrix $\left(\mu_{j k}\right)_{j, k \in \mathbf{N}}$ with real entries is said to be bounded if there exists a constant $M$ such that

$$
\left|\sum_{j=1}^{\infty} \sum_{k=1}^{\infty} \mu_{j k} x_{j} x_{k}\right| \leq M
$$

for every sequence $\left(x_{k}\right)_{k \in \mathbf{N}} \in \ell^{2}$. Equivalently (see [8, p. 301;11, p. 571]), the matrix is bounded if for every $\left(x_{k}\right)_{k \in \mathbf{N}} \in \ell^{2}$ the sequence $\left(y_{k}\right)_{k \in \mathbf{N}}$, where

$$
y_{j}=\sum_{k=1}^{\infty} \mu_{j k} x_{k} \text {, }
$$

also belongs to $\ell^{2}$.

Now denote by $(j, k)$ the greatest common divisor of $j$ and $k$.

Lemma 4 [11, pp. 577-578]. The infinite matrix

$$
\left(\frac{(j, k)^{2 \sigma}}{(j k)^{\sigma}}\right)_{j, k \in \mathbf{N}}
$$

is bounded if and only if $\sigma>1$.

With this we are ready to prove

Lemma 5. The transformation

$$
b_{n}=\sum_{j=1}^{\infty} \frac{a_{n j}}{j^{\sigma}} \quad\left(\sigma>\frac{1}{2}\right)
$$

maps every sequence $\left(a_{n}\right)_{n \in \mathbf{N}} \in \ell^{2}$ into a sequence $\left(b_{n}\right)_{n \in \mathbf{N}} \in \ell^{2}$ if and only if $\sigma>1$.

Proof. Using the Cauchy-Schwarz inequality, it is readily seen that $b_{n}$ exists for all $\sigma>1 / 2$. For $N \in \mathbf{N}$, we may write

$$
\begin{aligned}
B_{N}: & =\sum_{n=1}^{N}\left|b_{n}\right|^{2}=\sum_{n=1}^{N} \sum_{j=1}^{\infty} \sum_{k=1}^{\infty} \frac{a_{n j}}{j^{\sigma}} \cdot \frac{\bar{a}_{n k}}{k^{\sigma}} \\
& =\sum_{n=1}^{N} \sum_{j=1}^{\infty} \sum_{k=1}^{\infty} \frac{a_{n j}}{(n j)^{\sigma}} \cdot \frac{\bar{a}_{n k}}{(n k)^{\sigma}} \cdot n^{2 \sigma} \\
& =\sum_{j=1}^{\infty} \sum_{k=1}^{\infty} a_{j} \bar{a}_{k} \frac{1}{j^{\sigma}} \cdot \frac{1}{k^{\sigma}} \sum_{d \leq N, d \mid(j, k)} d^{2 \sigma},
\end{aligned}
$$

where the last summation extends to all divisors $d$ of $(j, k)$ that do not exceed $N$. Hence $B_{N}$ is the value of the quadratic form

$$
\sum_{j=1}^{\infty} \sum_{k=1}^{\infty} c_{j k}(N, \sigma) a_{j} \bar{a}_{k}
$$


with the nonnegative coefficients

$$
c_{j k}(N, \sigma)=\frac{1}{(j k)^{\sigma}} \sum_{d \leq N, d \mid(j, k)} d^{2 \sigma} .
$$

For $\sigma>1$, we find

$$
\begin{aligned}
c_{j k}(N, \sigma) & \leq \frac{1}{(j k)^{\sigma}} \sum_{d \mid(j, k)} d^{2 \sigma}=\frac{(j, k)^{2 \sigma}}{(j k)^{\sigma}} \sum_{\nu=1}^{(j, k)} \frac{1}{\nu^{2 \sigma}} \\
& \leq \frac{\pi^{2}}{6} \cdot \frac{(j, k)^{2 \sigma}}{(j k)^{\sigma}} .
\end{aligned}
$$

On the other hand, for $1 / 2<\sigma \leq 1$ we get

$$
c_{j k}(N, \sigma) \geq \frac{(j, k)^{2 \sigma}}{(j k)^{\sigma}} \quad \text { if } N \geq \max \{j, k\} .
$$

The estimates (6) and (7) in conjunction with Lemma 4 show that for all $\left(a_{n}\right)_{n \in \mathbf{N}} \in \ell^{2}$ the corresponding $B_{N}$ remains bounded as $N \rightarrow \infty$, if and only if $\sigma>1$.

\section{PROOFS OF THE THEOREMS}

Proof of Theorem 1. If $f \in \mathscr{T}_{k}$, then $f_{h} \in \mathscr{T}_{k}$ for all $h \in \mathbf{R}$ and hence $R_{n}\left[f_{h}\right]=$ 0 for all $n>k$ as indicated in the introduction.

Now suppose that $R_{n}\left[f_{h}\right]=0$ for all $n>k$ and $|h| \leq \pi / n$, Then, given $\varepsilon>0$ and $n>k$, there exists by Lemma 2 an $m_{0} \in \mathbf{N}$ such that

$$
\left|2 \pi \sum_{|j|<[m / n]}^{\prime}\left(1-\frac{|j n|}{m+1}\right) f^{-}(j n) e^{i j n h}\right|<\varepsilon
$$

for all $m \geq m_{0}$ and $|h| \leq \pi / n$. Hence the trigonometric polynomial

$$
t(\theta)=2 \pi \sum_{|j| \leq[m / n]}^{\prime}\left(1-\frac{|j n|}{m+1}\right) f^{-}(j n) e^{i j \theta}
$$

is bounded in absolute value by $\varepsilon$ for all $\theta \in \mathbf{R}$ if $m \geq m_{0}$. In particular,

$$
\left|2 \pi\left(1-\frac{n}{m+1}\right) f^{-}( \pm n)\right|<\varepsilon \text { for } m \geq m_{0}, n>k .
$$

From this we readily conclude that $f \in \mathscr{T}_{k}$.

Proof of Theorem 2. Let $f \in B_{2 \pi, d}^{2}$. According to Lemma 3, $f^{-}(n)=$ $a_{n} e^{-|n| d}$, where $\left(a_{n}\right)_{n \in \mathbf{N}} \in \ell^{2}$. Hence, in view of Lemma 2, we obtain for all $n \in \mathbf{N}$ and $h \in \mathbf{R}$

$$
\left|R_{n}\left[f_{h}\right]\right| \leq 2 \pi \sum_{j \in \mathbf{Z}}^{\prime}\left|a_{j n}\right| e^{-n|j| d}:=b_{n} e^{-n d} .
$$


Using the Cauchy-Schwarz inequality we find

$$
\left|b_{n}\right|^{2} \leq \frac{8 \pi^{2}}{1-e^{-d}} \sum_{j \in \mathbf{Z}}^{\prime}\left|a_{j n}\right|^{2} e^{-n(|j|-1) d},
$$

and hence, for all $N \in \mathbf{N}$,

$$
\begin{aligned}
\sum_{n=1}^{N}\left|b_{n}\right|^{2} & \leq \frac{8 \pi^{2}}{1-e^{-d}} \sum_{\ell \in \mathbf{Z}}^{\prime}\left|a_{\ell}\right|^{2} \sum_{\nu=0}^{|\ell|-1} e^{-\nu d} \\
& \leq \frac{8 \pi^{2}}{\left(1-e^{-d}\right)^{2}} \sum_{\ell \in \mathbf{Z}}^{\prime}\left|a_{\ell}\right|^{2}
\end{aligned}
$$

which means that (i) implies (ii).

Now let us assume that (ii) holds, and let $n$ be an arbitrary but fixed positive integer. Then by Lemma 2 there exists an $m_{0} \in \mathbf{N}$ such that, for all $m \geq m_{0}$,

$$
\left|2 \pi \sum_{|j| \leq[m / n]}^{\prime}\left(1-\frac{|j n|}{m+1}\right) f^{-}(j n) e^{i j n h}\right| \leq\left(b_{n}+\frac{1}{n}\right) e^{-n d} .
$$

Hence the right-hand side is a bound for the coefficients of the trigonometric polynomial (8); in particular,

for all $m \geq m_{0}$; i.e.,

$$
\left|2 \pi\left(1-\frac{n}{m+1}\right) f^{-}( \pm n)\right| \leq\left(b_{n}+\frac{1}{n}\right) e^{-n d}
$$

$$
\left|f^{-}( \pm n)\right| \leq \frac{1}{2 \pi}\left(b_{n}+\frac{1}{n}\right) e^{-n d}
$$

Finally, with the help of Lemma 3, we see that $f$ is the restriction to $\mathbf{R}$ of a function belonging to $B_{2 \pi, d}^{2}$.

Proof of Theorem 3. According to a well-known result [3, Theorem 4.1.10], statement (i) is equivalent to

$$
f^{-}(n)=(\text { in })^{-k} c_{n} \text { for all } n \in \mathbf{Z} \text {, where }\left(c_{ \pm n}\right)_{n \in \mathbf{N}} \in \ell^{2} \text {. }
$$

Hence, by Lemma 2,

$$
\left|R_{n}\left[f_{h}\right]\right| \leq 2 \pi \sum_{j \in \mathbf{Z}}^{\prime}\left|c_{j n}\right| /|j n|^{k}=: b_{n} n^{-k}
$$

for all $n \in \mathbf{N}$ and $h \in \mathbf{R}$. If $k \geq 2$ and $M_{k}:=\sum_{j \in \mathbf{N}} j^{-k}$, we obtain, using the Cauchy-Schwarz inequality,

$$
\left|b_{n}\right|^{2} \leq 8 \pi^{2} \sum_{j \in \mathbf{Z}}^{\prime}|j|^{-k}\left|c_{j n}\right|^{2} \quad \text { for } n \in \mathbf{N}
$$

Consequently,

$$
\sum_{n=1}^{N}\left|b_{n}\right|^{2} \leq 8 \pi^{2} M_{k} \sum_{\ell \in \mathbf{Z}}^{\prime}\left|c_{\ell}\right|^{2} \sum_{j=1}^{\ell} j^{-k} \leq 8 \pi^{2} M_{k}^{2} \sum_{\ell \in \mathbf{Z}}\left|c_{\ell}\right|^{2},
$$

and (ii) follows. 
Now suppose that (ii) holds. For every $n \in \mathbf{N}$ there corresponds by Lemma 2 an $m_{0} \in \mathbf{N}$ such that

$$
\left|2 \pi \sum_{|j| \leq[m / n]}^{\prime}\left(1-\frac{|j n|}{m+1}\right) f^{-}(j n) e^{i j n h}\right| \leq\left(b_{n}+\frac{1}{n}\right) n^{-k}
$$

for all $m>m_{0}$ and $|h| \leq \pi / n$. Considering again the trigonometric polynomial $(8)$, we find that $\left(i^{*}\right)$ holds which is, of course, equivalent to (i).

Proof of Theorem 4. By Lemma 5, there exists a sequence $\left(a_{n}\right)_{n \in \mathbf{N}} \in \ell^{2}$ such that, for

$$
b_{n}:=\sum_{j=1}^{\infty} \frac{a_{n j}}{j}
$$

we have $\sum_{n=1}^{\infty}\left|b_{n}\right|^{2}=\infty$. Defining

$$
f(x):=\sum_{n=1}^{\infty} \frac{a_{n}}{n} e^{i n x},
$$

we obtain a function $f \in W_{L^{2}}^{1}$ for which, by Lemma 2,

$$
n R_{n}[f]=-2 \pi \sum_{j=1}^{\infty} \frac{a_{n j}}{j}=-2 \pi b_{n} .
$$

This leads immediately to the desired result.

\section{REFERENCES}

1. G. R. Blakely, I. Borosh, and C. K. Chui, A two-dimensional mean problem, J. Approx. Theory 22 (1971), 11-26.

2. I. Borosh and C. K. Chui, On characterization of functions by their Gauss-Chebyshev quadratures, SIAM J. Math. Anal. 10 (1979), 532-541.

3. P. L. Butzer and R. J. Nessel, Fourier analysis and approximation, vol. 1, Birkhäuser, Basel-Stuttgart, 1971.

4. C. H. Ching, On averages of a function and its application to boundary value problems with insufficient data, SIAM J. Math. Anal. 5 (1974), 941-947.

5. C. H. Ching and C. K. Chui, Analytic functions characterized by their means on an arc, Trans. Amer. Math. Soc. 184 (1973), 176-183.

6. $\ldots$, Mean boundary value problems and Riemann series, J. Approx. Theory 10 (1974), 324-336.

7. C. K. Chui and P. W. Smith, Characterization of a function by certain infinite series it generates, Pacific J. Math. 53 (1974), 363-371.

8. E Hellinger and O. Toeplitz, Grundlagen für eine Theorie der unendlichen Matrizen, Math. Ann. 69 (1910), 289-330.

9. J. H. Loxton and J. W. Sanders, The kernel of a rule of approximate integration, J. Austral. Math. Soc. Ser. B 21 (1980), 257-267.

10. G. Meinardus, Approximation of functions: theory and numerical methods, Springer, New York, 1967.

11. A Wintner, Diophantine approximations and Hilbert's space, Amer. J. Math. 66 (1944), 564-578. 
12. A. A. Žensykbaev, Best quadrature formula for the class $W_{L^{2}}^{r}$, Analysis Mathematica 3 (1977), 83-95.

Département de Mathématiques et de Statistique, Université de Montréal, Montréal, Québec H3C 3J7, Canada

Mathematisches Institut, Universität ERlangen-Nürnberg, Bismarckstrasse $1 \frac{1}{2}$, D8520 ERLANGEN, Germany 